\title{
ESTIMATION DES EFFETS DE PROXIMITÉ DANS LE PROCESSUS DE CONVERGENCE RÉGIONALE : UNE APPROCHE PAR L'ÉCONOMÉTRIE SPATIALE SUR 92 RÉGIONS EUROPÉENNES (1980-1995) \\ Catherine Baumont, Cem Ertur, Julie Le Gallo
}

Armand Colin | « Revue d'Économie Régionale \& Urbaine »

2002/2 avril | pages 203 à 216

ISSN 0180-7307

Article disponible en ligne à l'adresse :

http://www.cairn.info/revue-d-economie-regionale-et-urbaine-2002-2-page-203.htm

\section{Pour citer cet article :}

Catherine Baumont et al., «Estimation des effets de proximité dans le processus de convergence régionale : une approche par l'économétrie spatiale sur 92 régions européennes (1980-1995) », Revue d'Économie Régionale \& Urbaine 2002/2 (avril), p. 203-216.

DOI 10.3917/reru.022.0203

Distribution électronique Cairn.info pour Armand Colin.

(C) Armand Colin. Tous droits réservés pour tous pays.

La reproduction ou représentation de cet article, notamment par photocopie, n'est autorisée que dans les limites des conditions générales d'utilisation du site ou, le cas échéant, des conditions générales de la licence souscrite par votre établissement. Toute autre reproduction ou représentation, en tout ou partie, sous quelque forme et de quelque manière que ce soit, est interdite sauf accord préalable et écrit de l'éditeur, en dehors des cas prévus par la législation en vigueur en France. Il est précisé que son stockage dans une base de données est également interdit. 


\title{
ESTIMATION DES EFFETS DE PROXIMITÉ DANS LE PROCESSUS DE CONVERGENCE RÉGIONALE : UNE APPROCHE PAR L'ÉCONOMÉTRIE SPATIALE SUR 92 RÉGIONS EUROPÉENNES (1980-1995)*
}

\section{AN ESTIMATION OF PROXIMITY EFFECTS IN THE REGIONAL CONVERGENCE PROCESS : A SPATIAL ECONOMETRICS APPROACH ON 92 EUROPEAN REGIONS (1980-1995)}

\author{
par \\ Catherine BAUMONT \\ Professeur \\ Catherine.baumont@u-bourgogne.fr \\ Cem ERTUR \\ Maître de Conférences \\ Cem.ertur@u-bourgogne.fr \\ Julie LE GALLO \\ ATER \\ Julie.Le-Gallo@u-bourgogne.fr \\ Université de Bourgogne \\ LATEC - Pôle d'Économie et de Gestion \\ B.P. 26611 \\ F - 21066 DIJON CEDEX
}

Mots-clés : Convergence, économétrie spatiale, effets de débordement.

Key-words : Convergence, spatial econometrics, spillover effects.

Classification JEL : C51, R11, R15.

\footnotetext{
* Première version février 2001, version révisée octobre 2001.

Les auteurs remercient J.M. HURIOT, C. LACOUR et B. SCHMITT pour leurs commentaires et suggestions. Les auteurs restent seuls responsables des insuffisances que pourrait comporter ce texte.
} 
204 Estimation des effets de proximité dans le processus de convergence régionale

\section{- INTRODUCTION -}

Un des thèmes dominants traités dans la littérature macroéconomique depuis une dizaine d'années est celui de la convergence des économies nationales ou régionales (BAUMOL, 1986 ; BARRO et SALA-I-MARTIN, 1991, 1995).

De nombreux travaux empiriques cherchant à tester cette hypothèse à l'échelle internationale ou régionale se heurtent à des difficultés économétriques variées (problèmes d'hétérogénéité, de variables omises, d'erreurs de mesure ...) rendant délicate l'interprétation des résultats (TEMPLE, 1999 ; ISLAM, 1998). En outre, la dimension géographique du phénomène de convergence est largement ignorée alors qu'elle soulève deux réflexions immédiates : d'une part, certains facteurs explicatifs de la convergence économique tels que la diffusion des technologies et la mobilité des facteurs ont une forte dimension spatiale et d'autre part, les territoires étudiés sont liés entre eux par leur position géographique. On observe ainsi que les performances économiques de régions voisines sont similaires. Une conséquence importante de la dépendance géographique est que l'inférence statistique basée sur des estimations d'un modèle par les Moindres Carrés Ordinaires (MCO) n'est plus fiable.

Il semblerait alors naturel que les études empiriques de la convergence se préoccupent du problème de la dépendance spatiale entre les zones géographiques étudiées et une manière de prendre en compte ce problème est de considérer l'auto-corrélation spatiale (CLIFF et ORD, 1981 ; LE GALLO, 2001). Pourtant, depuis DE LONG et SUMMERS (1991) qui avaient soulevé la question de la dépendance spatiale (1)* sans arriver à la traiter de manière satisfaisante, seules quelques études récentes intègrent le rôle des effets spatiaux dans les processus de convergence (MORENO et TREHAN, 1997 ; FINGLETON, 1999 ; REY et MONTOURI, 1999).

L'objectif de cet article est d'intégrer les effets de la dépendance spatiale dans l'estimation de la convergence des économies régionales. Nous nous intéressons plus précisément au rôle joué par la proximité géographique dans le processus de croissance régionale. En utilisant les outils de l'économétrie spatiale (ANSELIN, 1988 ; ANSELIN et FLORAX, 1995), nous estimons un modèle de $\beta$-convergence sur un ensemble de 92 régions européennes contiguës pour la période 1980-1995. Nous détectons la présence d'auto-corrélation spatiale dans le modèle de $\beta$-convergence absolue ce qui met en doute les résultats de linférence statistique fondée sur les MCO. En utilisant les spécifications économétriques appropriées, nous pouvons ensuite estimer les effets de débordement géographiques liés à la proximité des régions et nous montrons que le taux de croissance d'une région donnée est influencé par les taux de croissance des régions voisines.

* Les chiffres entre parenthèses renvoient aux notes en fin d'article. 


\section{- I - \\ EFFETS SPATIAUX ET MODÈLES DE $\beta$-CONVERGENCE}

Le concept de convergence fondé sur les modeles de croissance néoclassique (SOLOW, 1956) signifie, qu'à long terme, le taux de croissance du revenu ou de la production par tête d'une économie tend vers un état régulier et ceci indépendamment des conditions initiales dans lesquelles se trouvait cette économie. A l'état régulier, le taux de croissance est égal au taux de progrès technique, supposé identique pour toutes les économies. L'estimation du modele de $\beta$-convergence (BARRO et SALA-I-MARTIN, 1995) en coupe transversale sert habituellement à évaluer la pertinence de ce concept de convergence. Cependant, puisque l'on étend la propriété du modèle néoclassique, définie pour une économie, à un ensemble de plusieurs économies, il convient de distinguer si on est en présence d'économies aux caractéristiques identiques ou différentes. Ces caractéristiques déterminent en effet l'état régulier du PIB par tête. Ainsi, la $\beta$ convergence peut être absolue (inconditionnelle) ou conditionnelle. Elle est absolue lorsque les économies sont structurellement identiques et ont donc le même état régulier : dans ce cas, les économies, même si elles sont caractérisées par des conditions initiales différentes, progresseront vers le même état régulier. En revanche, la $\beta$-convergence est conditionnelle lorsque les économies sont différentes et elles convergeront alors vers leur propre état régulier. Ce dernier cas correspond en fait au cadre d'hypothèses du modèle de croissance néoclassique. Par conséquent, si les économies convergent vers des états réguliers différents, le phénomène de rattrapage des économies n'est absolument pas garanti, même si certaines économies « pauvres » croissent plus vite que certaines économies « riches». Ce n'est que dans le cas de la $\beta$-convergence absolue que les prédictions en termes de rattrapage sont valides.

\subsection{Les modèles de $\beta$-convergence}

L'hypothèse de $\beta$-convergence absolue est habituellement testée sur le modèle en coupe transversale suivant :

$$
g_{T}=\alpha S+\beta y_{0}+\varepsilon \quad \varepsilon \sim N\left(0, \sigma_{\varepsilon}^{2} I\right)
$$

où $g_{T}$ est le vecteur $(n \times l)$ des taux de croissance moyens du PIB par tête entre les dates 0 et $T, y_{0}$ est le vecteur des niveaux de PIB par tête à la date $0, T$ est la longueur de la période d'étude, $\alpha$ et $\beta$ sont des paramètres inconnus à estimer, $S$ est le vecteur unité et $\varepsilon$ est le vecteur des erreurs avec les propriétés usuelles; les valeurs des PIB par tête étant exprimées en logarithmes.

On dit qu'il y a $\beta$-convergence lorsque $\beta$ est négatif et statistiquement significatif puisque dans ce cas le taux de croissance moyen du PIB par tête entre les dates 0 et $T$ est négativement corrélé avec le niveau initial du PIB par tête. L'estimation de $\beta$ permet de calculer la vitesse de convergence : $\theta=-\ln (1+T \beta) / T$, et la durée nécessaire pour que les économies comblent la 
206 Estimation des effets de proximité dans le processus de convergence régionale

moitié de l'écart qui les sépare de leur état régulier, appelée la demivie : $\tau=-\ln (2) / \ln (1+\beta)$.

Le test de l'hypothèse de $\beta$-convergence conditionnelle repose quant à lui sur l'estimation du modèle suivant où l'on doit isoler les variables qui différencient les régions :

$$
g_{T}=\alpha S+\beta y_{0}+X \Phi+\varepsilon \quad \varepsilon \sim N\left(0, \sigma_{\varepsilon}^{2} I\right)
$$

$X$ est une matrice de variables, permettant de maintenir constant l'état régulier de chaque économie, où l'on peut trouver des variables d'état, comme le stock de capital physique et le stock de capital humain, et des variables de contrôle ou d'environnement, comme le ratio de la consommation publique au $\mathrm{PIB}$, le ratio de l'investissement domestique au PIB, les modifications des termes de l'échange, le taux de fécondité, le degré d'instabilité politique etc. (BARRO et SALA-IMARTIN, 1995). Cette approche est largement utilisée dans les études internationales : elle est basée sur des spécifications exploratoires plus ou moins ad hoc (2) ou sur des spécifications formellement dérivées de modèles structurels de croissance à la suite de MANKIW, ROMER et WEIL (1992).

Une autre manière de tester l'hypothèse de convergence conditionnelle repose toujours sur l'équation /1/ mais elle est estimée sur des sous-échantillons d'économies pour lesquelles l'hypothèse d'états réguliers similaires semble acceptable. Cette approche est privilégiée par SALA-I-MARTIN (1996a, 1996b) lorsqu'il étudie la convergence régionale au sein de différents pays.

Il faut souligner cependant que dans les tests de convergence précédemment évoqués, l'analyse porte sur des régions observées en coupe transversale en supposant implicitement que chacune d'entre elles est une entité géographiquement indépendante et en ignorant la possibilité d'interactions spatiales. Or cette dimension spatiale des données devrait faire l'objet d'une attention particulière dans l'étude des processus de convergence d'autant plus que l'utilisation de données spatiales n'est ni neutre ni immédiate : elle nécessitera le traitement de l'auto-corrélation spatiale (3).

\subsection{Définition de l'auto-corrélation spatiale}

L'auto-corrélation spatiale (CLIFF et ORD, 1981) se réfère à l'absence d'indépendance entre observations et indique la corrélation d'une grandeur avec elle-même provenant de la disposition géographique des données. En d'autres termes, l'auto-corrélation spatiale positive se traduit par une tendance à la concentration dans l'espace de valeurs faibles ou élevées d'une variable aléatoire. En revanche, l'auto-corrélation spatiale négative signifie que chaque localisation tend à être entourée par des localisations voisines pour lesquelles la variable aléatoire prend des valeurs très différentes.

L'auto-corrélation spatiale a deux sources principales. Elle peut d'abord provenir du fait que les données sont affectées par des processus qui relient des lieux différents et qui sont à l'origine d'une organisation spatiale particulière des 
activités. Par exemple un phénomène tel que la diffusion technologique, implique que l'intensité de ce phénomène dépend de la distance à l'origine : des localisations proches les unes des autres et situées à des distances comparables de l'origine auront ainsi des valeurs similaires pour le phénomène étudié. Plus généralement, les événements ou les circonstances en un lieu donné affectent les conditions en d'autres lieux si ces derniers interagissent d'une manière ou d'une autre : par des mouvements de biens, de personnes, de capitaux, par des externalités spatiales ... soit plus généralement toutes les formes de comportements où un acteur économique réagit aux actions d'autres acteurs. Ces différents éléments sont ainsi à l'origine d'ordonnancements géographiques particuliers des phénomènes observés dans l'espace que la présence d'autocorrélation spatiale permet de détecter. L'auto-corrélation spatiale peut également provenir d'une mauvaise spécification du modèle, du fait de variables omises, d'une forme fonctionnelle incorrecte, d'erreurs de mesure sur les variables ou encore de problèmes d'agrégation.

$\mathrm{Ne}$ pas prendre en compte l'auto-corrélation spatiale, alors qu'elle est présente, produit des estimateurs inefficients et conduit à une inférence statistique peu fiable (ANSELIN, 1988). Pour y remédier, il faut utiliser les méthodes appropriées de l'économétrie spatiale. Par ailleurs, modéliser l'auto-corrélation spatiale permet aussi de capter le rôle de l'espace dans la formation des phénomènes étudiés, en particulier de capter les effets de débordement géographiques (LE GALLO, 2001).

\subsection{La dépendance spatiale en économie géographique}

Les formes prises par la répartition géographique des phénomènes économiques peuvent également être mises en perspective avec les prédictions des théories économiques spatiales. En effet, ces théories apportent les éléments pour comprendre les choix de localisation et les processus d'agglomération des activités économiques et montrent que des organisations spatiales particulières en découlent. Plus précisément, nous nous intéressons ici aux théories de la Nouvelle Économie Géographique (4), initiées par KRUGMAN (1991). Une première prédiction est la tendance à la concentration des activités économiques sur un nombre réduit de territoires. La répartition géographique des espaces riches en activités économiques peut alors être caractérisée par une dépendance spatiale puisque les lieux d'agglomération sont identifiés soit grâce à des conditions naturelles ou de première nature soit en fonction de conditions dites de seconde nature lorsque l'attractivité d'un lieu dépend des activités économiques qui y sont présentes. Par ailleurs, les processus d'agglomération apparaissent fortement cumulatifs : l'agglomération favorise l'agglomération. Ainsi, même si au départ il y a une répartition géographique homogène des activités économiques, un choc exogène, comme le choix d'une firme de se localiser en un lieu plutôt qu'en un autre, peut conduire à la formation d'une agglomération en ce lieu.

Une deuxième prédiction concerne les liens entre les processus d'agglomération, i.e. d'accumulation des activités économiques dans l'espace, et la croissance, i.e. l'accumulation des activités économiques dans le temps. En se basant sur la similarité des mécanismes économiques impliqués à la fois dans les 
208 Estimation des effets de proximité dans le processus de convergence régionale

processus de concentration spatiale et dans les processus d'accumulation temporelle de certaines activités économiques favorables à la croissance, telles que la production d'inputs ou de biens différenciés, la $R \& D$, les infrastructures publiques, l'innovation, les services aux entreprises..., on a pu considérer l'agglomération comme un facteur de croissance (BAUMONT, 1997). Plusieurs auteurs ont ainsi formalisé les liens complexes unissant les processus d'agglomération et les processus de croissance (ENGLMANN et WALZ, 1995 ; KUBO 1995 ; MARTIN et OTTAVIANO, 1999). Les résultats fournis par ces théories dites de la Synthèse Géographie-Croissance (BAUMONT et HURIOT, 1999) montrent d'une façon générale que d'une part, l'agglomération favorise la croissance, ce qui signifie que la répartition spatiale inégale des activités économiques est une configuration géographique efficace pour la croissance, et que d'autre part, la croissance peut favoriser la concentration géographique des activités économiques. L'élargissement des marchés, la diminution des coûts de transaction, l'accroissement de la mobilité du travail, le degré de développement des économies, les relations de «vertical linkage » entre les firmes ou encore l'existence d'effets de débordement sont autant d'éléments pouvant expliquer la force des interactions unissant les processus de croissance et d'agglomération.

Si on s'intéresse plus particulièrement aux effets de débordement, un résultat théorique important montre qu'il faut distinguer les effets de débordement locaux et les effets de débordement globaux. Les premiers signifient que seule la région dans laquelle les activités économiques sont concentrées bénéficie des avantages de la concentration. Dans ces conditions, les effets de débordement locaux renforcent les phénomènes de polarisation et de croissance inégale des espaces. En revanche, en présence d'effets de débordement globaux, toutes les régions peuvent bénéficier des avantages de la concentration en une région particulière. Dans ce cas, la répartition spatiale des activités entre les régions peut être moins inégalitaire, ce qui favorise la réduction des disparités de croissance entre les régions (ENGLMANN et WALZ, 1995). On peut enfin considérer des situations intermédiaires dans lesquelles la concentration des activités économiques dans chacune des régions peut produire à la fois des effets de débordement locaux et globaux d'intensités différentes (KUBO, 1995).

Face à ces différents résultats, incorporer les effets géographiques de débordement entre les régions apparaît comme une piste intéressante pour mieux comprendre les phénomènes de croissance et de convergence. L'approche empirique que nous allons réaliser grâce à l'étude de la dépendance spatiale entre les régions permet l'investigation de cette question. 
- II -

\section{ANALYSE ÉCONOMÉTRIQUE SPATIALE DE LA $\beta$-CONVERGENCE}

Nous utilisons les techniques de l'économétrie spatiale (CLIFF et ORD, 1981 ; ANSELIN, 1988 ; ANSELIN et FLORAX, 1995 ; LE GALLO, 2001) pour détecter et traiter l'auto-corrélation spatiale dans le modèle de $\beta$-convergence absolue sur les PIB par tête des régions européennes pour la période 1980-1995. Les données sont issues de la banque de données EUROSTAT-REGIO.

L'interconnexion spatiale entre les régions est captée à travers une matrice de poids qui est ici une matrice de contiguiité d'ordre 1 , notée $W$, où chaque terme est égal à 1 si les régions partagent une frontière commune, qu'elles appartiennent ou non au même pays, et 0 sinon. Ce choix correspond au contexte de l'intégration régionale dans lequel nous nous plaçons. Cette matrice de poids est ensuite standardisée pour permettre la comparaison des valeurs des coefficients entre les différents modèles estimés. Le choix de la matrice de poids nous conduit à retenir parmi l'ensemble des régions européennes, celles qui sont contiguës, c'est-à-dire celles qui partagent une ou plusieurs frontières communes. En conséquence, nous avons exclu le Royaume-Uni, l'Irlande, la Grèce et les îles. Au total notre échantillon comprend 92 régions (au niveau NUTS2).

Nous estimons d'abord le modèle de $\beta$-convergence absolue et effectuons différents tests visant à détecter la présence d'effets spatiaux. Nous estimons ensuite une spécification intégrant explicitement ces effets spatiaux.

\subsection{Estimation du modèle de $\beta$-convergence absolue}

Prenons comme point de départ le modèle de $\beta$-convergence absolue :

$$
g_{T}=\alpha S+\beta y_{1980}+\varepsilon \quad \varepsilon \sim N\left(0, \sigma_{\varepsilon}^{2} I\right)
$$

où $g_{T}$ est le vecteur de dimension $N=92$ des taux de croissance moyens des PIB par tête pour chaque région $i$ entre 1995 et $1980, T=15, y_{1980}$ est le vecteur contenant les observations des PIB par tête pour toutes les régions en 1980, $\alpha$ et $\beta$ sont les paramètres inconnus à estimer, $S$ est le vecteur unité et $\varepsilon$ est le vecteur des erreurs avec les propriétés habituelles; les valeurs des PIB par tête en ECU étant exprimées en logarithmes.

Les résultats de l'estimation par les moindres carrés ordinaires de ce modèle sont donnés dans le tableau 1. On note que les variables sont significatives et que le coefficient associé au niveau de PIB par tête est négatif, ce qui confirme l'hypothèse de convergence pour les régions européennes. La vitesse de convergence associée à cette estimation est de $1,70 \%$ et la demi-vie est de 46 ans. Ces résultats indiquent que le processus de convergence est faible et sont conformes aux autres études empiriques sur la convergence des régions européennes (BARRO et SALA-I-MARTIN, 1995 ; JEAN-PIERRE, 1999). 
210 Estimation des effets de proximité dans le processus de convergence régionale

Tableau 1 : Estimation par les MCO du modèle $/ 3 /$.

\begin{tabular}{|c|c|c|c|c|}
\hline constante & $\hat{\beta}$ & demi-vie & \multicolumn{2}{|c|}{ vitesse de convergence } \\
\hline 0,190 & $-0,015$ & 46 & \multicolumn{2}{|c|}{$\begin{array}{l}1,70 \% \\
(0,00)\end{array}$} \\
$(0,000)$ & $(0,000)$ & & \multicolumn{2}{|c|}{$\hat{\sigma}^{2}$} \\
\hline $\mathbf{R}^{2}$ & LIK & AIC & BIC & $\hat{\sigma}^{-5}$ \\
\hline 0,41 & 312,32 & $-620,64$ & $-615,59$ & $6,736.10^{-5}$ \\
\hline
\end{tabular}

Notes : Les probabilités critiques sont entre parenthèses. LIK : valeur de la fonction de vraisemblance à l'optimum. AIC : critère d'AKAIKE (1974). BIC : critère de SCHWARZ (1978).

En outre, cinq tests de l'auto-corrélation spatiale ont été effectués (5) : le test de MORAN (1948) adapté aux résidus d'une régression par CLIFF et ORD (1981). Ce test est très puissant contre les deux formes de dépendance spatiale mais ne permet pas de les discriminer (ANSELIN et FLORAX, 1995). Pour pouvoir les discriminer nous utilisons deux tests du multiplicateur de Lagrange ainsi que leur version robuste qui permettent de tester la présence des deux formes possibles de l'auto-corrélation spatiale (ANSELIN et alii, 1996) : LMERR pour une auto-corrélation spatiale des erreurs et LMLAG pour une variable auto-régressive ou endogène décalée. Les versions robustes RLMERR et RLMLAG ont une bonne puissance contre leur alternative spécifique. La variable endogène décalée est un vecteur de décalage spatial contenant les observations de la variable expliquée pré-multipliées par la matrice de poids $W$ : pour une région $i$ du vecteur $g_{T}$, la ligne correspondante du vecteur de décalage spatial $W g_{T}$ contient les moyennes pondérées des observations des régions contiguës à la région $i$.

La règle de décision suggérée par ANSELIN et FLORAX (1995) est utilisée pour déterminer la spécification la plus appropriée. Si LMLAG est plus significatif que LMERR et si RLMLAG est significatif alors que RLMERR ne l'est pas, alors le modèle approprié est le modèle spatial auto-régressif. Inversement, si LMERR est plus significatif que LMLAG et si RLMERR est significatif alors que RLMLAG ne l'est pas, alors le modèle approprié est le modèle avec erreurs spatialement auto-corrélées.

Les résultats des tests sont présentés dans le tableau 2. Le test de MORAN indique la présence d'une dépendance spatiale et la stratégie de test précédente conduit à retenir le modèle avec erreurs spatialement auto-corrélées.

Tableau 2 : Résultats des tests d'auto-corrélation spatiale.

\begin{tabular}{|c|c|c|c|c|}
\hline MORAN & LMERR & RLMERR & LMLAG & RLMLAG \\
\hline 5,074 & 21,249 & 5,227 & 16,184 & 0,162 \\
$(0,000)$ & $(0,000)$ & $(0,022)$ & $(0,000)$ & $(0,688)$ \\
\hline
\end{tabular}

Notes : Les probabilités critiques sont entre parenthèses. MORAN est le test de MORAN (1948) adapté aux résidus d'une régression (CLIFF et ORD, 1981). LMERR et LMLAG sont les tests du multiplicateur de Lagrange visant à tester respectivement la présence d'auto-corrélation spatiale des erreurs et d'une variable endogène décalée. RLMERR et RLMLAG sont leur équivalent robuste. 
Ainsi, le modele /3/ souffre d'une mauvaise spécification due à l'omission de l'auto-corrélation spatiale des erreurs. En réalité, chaque région n'est pas indépendante des autres, comme cela est fréquemment supposé dans les études antérieures effectuées au niveau régional. Le modèle de $\beta$-convergence absolue doit donc être modifié pour intégrer explicitement cette dépendance spatiale.

\subsection{Estimation du modèle à erreurs spatialement auto-corrélées}

Lorsque les erreurs suivent un processus spatial auto-régressif d'ordre 1, le modèle s'écrit :

$$
g_{T}=\alpha S+\beta y_{1980}+\varepsilon \quad \varepsilon=\lambda W \varepsilon+u \text { avec } u \sim N\left(0, \sigma^{2} I\right) \quad / 4 /
$$

$\lambda$ est le paramètre représentant l'intensité de l'auto-corrélation spatiale entre les résidus de la régression. Les erreurs n'étant pas indépendantes, l'utilisation des MCO dans ce cas produit des estimateurs centrés mais inefficients. Les résultats de l'estimation par le maximum de vraisemblance sont donnés dans le tableau 3.

Tableau 3 : Estimations par le maximum de vraisemblance du modèle $/ 4 /$.

\begin{tabular}{|c|c|c|c|c|}
\hline constante & $\hat{\beta}$ & $\hat{\lambda}$ & demi-vie & $\begin{array}{c}\text { vitesse de } \\
\text { convergence }\end{array}$ \\
\hline $\begin{array}{c}0,215 \\
(0,000)\end{array}$ & $\begin{array}{l}-0,017 \\
(0,000)\end{array}$ & $\begin{array}{c}0,601 \\
(0,000)\end{array}$ & 40 & $\begin{array}{r}1,96 \% \\
(0,000) \\
\end{array}$ \\
\hline LIK & AIC & BIC & $\hat{\sigma}^{2}$ & LMLAG* \\
\hline 323,57 & $-643,15$ & $-638,11$ & $4,66.10^{-5}$ & $\begin{array}{c}0,052 \\
(0,819) \\
\end{array}$ \\
\hline
\end{tabular}

Notes : Les probabilités critiques sont entre parenthèses. LIK : valeur de la fonction de vraisemblance à l'optimum. AIC : critère d'AKAIKE (1974). BIC : critère de SCHWARZ (1978). LMLAG* est le test du multiplicateur de Lagrange visant à tester la présence d'une variable endogène décalée dans le modèle avec auto-corrélation spatiale des erreurs.

Les coefficients sont tous significatifs. Le coefficient associé au niveau de PIB par tête initial est supérieur à celui du modèle / 3 / et l'estimation fait ressortir une forte auto-corrélation spatiale positive des erreurs $(\hat{\lambda}=0,601)$. Le test LMLAG* ne permet pas de rejeter l'hypothèse nulle d'absence d'une variable auto-régressive supplémentaire. Ce modèle est supérieur au précédent en termes de critères d'information (AKAIKE, 1974 ; SCHWARZ, 1978). Il apparaît donc que le modèle avec auto-corrélation spatiale des erreurs est la spécification la plus appropriée.

Cette spécification a deux implications. D'une part, la vitesse de convergence dans le modèle avec auto-corrélation spatiale est de $1,96 \%$ et est donc plus élevée que celle du modèle initial, la demi-vie n'est plus que de 40 ans. Ce résultat suggère que les effets de proximité, exprimés ici en termes de contiguïté, favorisent légèrement le processus de convergence régionale mais que celui-ci reste encore relativement faible. Cette première implication peut sembler qualitativement négligeable, mais nous devons souligner qu'il s'agit là de la seule manière appropriée d'estimer un modèle de $\beta$-convergence si l'on a détecté une 
212 Estimation des effets de proximité dans le processus de convergence régionale

auto-corrélation spatiale. C'est aussi la seule manière de réaliser des tests d'inférence statistique fiables.

D'autre part, l'auto-corrélation spatiale des erreurs implique qu'un choc aléatoire dans une région spécifique se propage à toutes les régions de l'échantillon (REY et MONTOURI, 1999).

En effet, puisque : $\varepsilon=\lambda W \varepsilon+u$, alors $\varepsilon=(I-\lambda W)^{-1} u$

et le modèle /4/ s'écrit :

$$
g_{T}=\alpha S+\beta y_{1980}+(I-\lambda W)^{-I} u
$$

De cette expression, on déduit qu'un choc aléatoire dans une région spécifique n'affecte pas seulement le taux de croissance de cette région, mais, à travers la transformation spatiale $(I-\lambda W)^{-1}$, a également un impact sur les taux de croissance des autres régions. De plus, même si une région quelconque a un nombre limité de voisins, l'opérateur inverse dans la transformation définit une covariance des erreurs diffusant des chocs spécifiques non seulement à ses voisins mais également à tout le système.

\subsection{Les effets de débordement}

Il faut souligner que le modèle /4/ peut encore se réécrire sous une autre forme qui peut s'interpréter comme un modèle de convergence conditionnelle intégrant des variables d'environnement spatial, définies dans cette étude en termes de contiguïté.

En effet, notons que :

$$
\varepsilon=g_{T}-\alpha S-\beta y_{1980}
$$

en pré-multipliant par $\lambda W$, on obtient :

$$
\lambda W \varepsilon=\lambda W g_{T}-\lambda \alpha W S-\lambda \beta W y_{1980}
$$

Comme :

$$
\varepsilon-\lambda W \varepsilon=u
$$

Alors :

$$
g_{t}=\alpha(I+\lambda W) S+\beta y_{1980}+\lambda W g_{T}+\gamma W y_{1980}+u
$$

Avec :

$$
\gamma=-\lambda \beta
$$

Le modèle /8/ est appelé modèle spatial de DURBIN contraint et peut être estimé par la méthode du maximum de vraisemblance. La restriction non-linéaire 19/ peut être testée par le test du facteur commun (BURRIDGE, 1981). Si la restriction $\gamma+\lambda \beta=0$ ne peut pas être rejetée, alors le modèle /8/ est équivalent au modèle $/ 4 /$. 
Ce modèle fait apparaître deux types d'effets de débordement. D'une part, le taux de croissance d'une région $i$ est influencé par le taux de croissance des régions contiguës à cette région, par le biais de la variable endogène décalée $W g_{T}$. La valeur du coefficient $\lambda$ associé indique l'intensité des effets de débordement géographique liés au rythme de croissance des régions. D'autre part, le taux de croissance d'une région $i$ est également influencé par les PIB par tête initiaux des régions contiguës, par le biais de la variable exogène décalée $W y_{1980}$. L'intensité des effets de débordement géographiques liés à la situation initiale des régions est indiquée par le paramètre associé $\gamma$.

Le test du facteur commun de BURRIDGE (1981) ne nous permet pas de rejeter la restriction $/ 9 /$, nous n'estimons donc pas le modèle spatial de DURBIN et l'estimation du modele /4/ permet alors de quantifier ce double effet de débordement : le taux de croissance dans une région est influencé de manière positive $(\hat{\lambda}=0,601)$ et significative par le taux de croissance des régions contiguës. En revanche, l'influence des PIB par tête initiaux des régions contiguës $(\hat{\gamma}=-\hat{\lambda} \hat{\beta}=0,0102)$ n'est pas significative (6).

Cet effet de débordement indique que les schémas d'association spatiale ne sont pas neutres quant aux performances économiques des régions. Plus une région est entourée de régions dynamiques et plus son taux de croissance est élevé. En d'autres termes, l'environnement géographique compte ce qui rejoint certaines prédictions des modèles de la Nouvelle Économie Géographique.

Par ailleurs, la réécriture du modèle intégrant l'auto-corrélation spatiale des erreurs aboutit à un modèle de convergence conditionnelle intégrant les variables d'environnement spatial qui permettent de contrôler dans cet article les effets de proximité spatiale de chaque région européenne dans le processus de la convergence régionale.

\section{- CONCLUSION -}

L'objectif de cet article est d'examiner les conséquences de la dépendance spatiale dans l'estimation du modèle de $\beta$-convergence et d'estimer l'impact de la proximité géographique sur la croissance régionale et le processus de convergence.

Nous détectons ainsi la présence d'une auto-corrélation spatiale dans un modèle de $\beta$-convergence inconditionnelle estimé sur un échantillon de 92 régions européennes pour la période 1980-1995. Les estimateurs des MCO sont alors inefficients et conduisent à une inférence statistique peu fiable. Les méthodes de l'économétrie spatiale nous orientent vers la spécification la plus appropriée : le modèle avec erreurs spatialement auto-corrélées. Cette spécification révèle que le processus de convergence est légèrement plus rapide. Par ailleurs, la re-formulation de cette spécification aboutit à un modèle de convergence conditionnelle intégrant des variables d'environnement spatial ce qui permet de faire apparaître un effet de débordement significatif. En effet, le taux 
214 Estimation des effets de proximité dans le processus de convergence régionale

de croissance d'une région donnée est positivement influencé par le taux de croissance des régions contiguës. Cette spécification implique également qu'un choc aléatoire dans une région donnée se propage à toutes les régions de l'échantillon.

Finalement ces résultats montrent que l'introduction de l'espace dans l'analyse de la convergence économique entre régions n'est pas neutre car les performances économiques d'une région sont en partie dépendantes de son environnement géographique. Mais, si les effets de proximité spatiale liés à la contiguïté sont ici soulignés, d'autres formes de dépendance spatiale pourraient également être testées comme celles liées à la distance ou à l'appartenance à des réseaux.

\section{BIBLIOGRAPHIE}

AKAIKE H., 1974, «A New Look at the Statistical Model Identification », IEEE Transactions on Automatic Control, AC-19, pp. 716-723.

ANSELIN L., 1988, Spatial Econometrics : Methods and Models, Dordrecht, Kluwer Academic Publishers.

ANSELIN L., 1999, SpaceStat, a software package for the analysis of spatial data, Version 1.90. Ann Arbor, BioMedware.

ANSELIN L., BERA A.K., FLORAX R., YOON M.J, 1996, « Simple Diagnostic Tests for Spatial Dependence », Regional Science and Urban Economics, n 26, pp. 77-104.

ANSELIN L., FLORAX R. Eds., 1995, New Directions in Spatial Econometrics, Berlin, Springer.

BARRO R.J., SALA-I-MARTIN X., 1991, « Convergence across States and Regions », Brookings Papers on Economic Activity, ${ }^{\circ} 1$, pp. 107-182.

BARRO R.J., SALA-I-MARTIN X., 1995, Economic Growth Theory, MIT Press.

BAUMOL. W.J., 1986, «Productivity Growth, Convergence and Welfare : What the Long-Run Data Show », American Economic Review, n 76, pp. 1072-1085.

BAUMONT C., 1997, "Croissance endogène des régions et espace », in CELIMENE F., LACOUR C. Eds., L'intégration régionale des espaces, Paris, Économica Bibliothèque de Science Régionale, pp. 33-61.

BAUMONT C., HURIOT J.-M., 1999, «L'interaction agglomération-croissance en économie géographique », in BAILLY A., HURIOT J.-M. Eds., Villes et Croissance : Théories, Modèles, Perspectives, Anthropos, pp. 133-168.

BURRIDGE P., 1981, «Testing for a Common Factor in a Spatial Autoregressive Model », Environment and Planing $A, \mathrm{n}^{\circ} 13$, pp. 795-800.

CLIFF A.D., ORD J.K., 1981, Spatial Processes : Models and Applications, Londres, Pion. 
DE LONG J.B., SUMMERS L.H., 1991, «Equipment, Investment and Economic Growth », Quarterly Journal of Economics, 106, 2, pp. 445-502.

ENGLMANN F.C., WALZ U., 1995, « Industrial Centers and Regional Growth in the Presence of Local Inputs », Journal of Regional Science, $\mathrm{n}^{\circ} 35$, pp. 3-27.

FINGLETON B., 1999, «Estimates of Time to Convergence : An Analysis of Regions of European Union », International Regional Science Review, $\mathrm{n}^{\circ} 22$, pp. 5-34.

FLORAX R., FOLMER H., 1992, «Specification and Estimation of Spatial Linear Regression Models », Regional Science and Urban Economics, $\mathrm{n}^{\circ} 22$, pp. 405-432.

FUJITA M., KRUGMAN P. et VENABLES A., 1999, The Spatial Economy, MTT Press, Cambridge.

FUJITA M., THISSE J., 1997, «Économie géographique. Problèmes anciens et nouvelles perspectives », Annales d'Économie et Statistique, $n^{\circ} 45 / 46$, pp. 37-87.

ISLAM N., 1998, «Convergence : Variation in Concept and Empirical Results », Working Paper, Department of Economics, Emory University.

JEAN-PIERRE P., 1999, «La convergence régionale européenne : une approche empirique par les clubs et les panels », Revue d'Économie Régionale et Urbaine, $\mathrm{n}^{\circ} 1$, pp. 21-44.

KRUGMAN P., 1991, «Increasing Returns and Economic Geography », Journal of Political Economy, n ${ }^{\circ} 99$, pp. 483-499.

KUBO Y., 1995, «Scale Economies, Regional Externalities, and the Possibility of Uneven Development », Journal of Regional Science, $\mathrm{n}^{\circ} 35$, pp. 29-42.

LE GALLO J., 2001, « Économétrie spatiale : l'auto-corrélation dans les modèles de régression linéaire », Économie et Prévision, à paraître.

LEVINE R., RENELT D., 1992, «A Sensitivity Analysis of Cross-Country Growth Regressions », American Economic Review, 82, 4, pp. 942-963.

MANKIW N.G., 1995, «The Growth of Nations », Brookings Papers on Economic Activity, $\mathrm{n}^{\circ}$ 1, pp. 275-326.

MANKIW N.G., ROMER D., WEIL D.N., 1992, « A Contribution to the Empirics of Economic Growth », Quarterly Journal of Economics, 107, 2, pp. 407-437.

MARTIN P., OTTAVIANO G.I.P., 1999, « Growing Locations: Industry Location in a Model of Endogenous Growth », European Economic Review, n 43, pp. 281-302.

MORAN P., 1948, «The Interpretation of Statistical Maps», Journal of the Royal Statistical Society, 10B, pp. 243-251.

MORENO R., TREHAN B., 1997, «Location and the Growth of Nations », Journal of Economic Growth, 2, 4, p. 399-418. 
216 Estimation des effets de proximité dans le processus de convergence régionale

REY S.J., MONTOURI B.D., 1999, «U.S. Regional Income Convergence: a Spatial Econometric Perspective », Regional Studies, ${ }^{\circ} 33$, pp. 145-156.

SALA-I-MARTIN X., 1996a, « Regional Cohesion: Evidence and Theories of Regional Growth and Convergence », European Economic Review, n ${ }^{\circ} 40$, pp. 1325-1352.

SALA-I-MARTIN X., 1996b, « The Classical Approach to Convergence Analysis », Economic Journal, n $^{\circ} 106$, pp. 1019-1036.

SCHWARZ G., 1978, «Estimating the Dimension of a Model », The Annals of Statistics, ${ }^{\circ}$ 6, pp. 461-464.

SOLOW R.M., 1956, «A Contribution to the Theory of Economic Growth », Quarterly Journal of Economics, $\mathrm{n}^{\circ} 70$, pp. 65-94.

TEMPLE J., 1999, «The New Growth Evidence», Journal of Economic Literature, $\mathrm{n}^{\circ} 7$, pp. 112-156.

\section{$R \hat{E} S U M \boldsymbol{E}^{\prime}$}

L'objectif de cet article est d'examiner les conséquences de la dépendance spatiale sur la croissance régionale et le processus de convergence. Sur un échantillon de 92 régions européennes sur la période 1980-1995, nous montrons que le modèle de $\beta$-convergence absolue doit être re-spécifié en raison de la présence d'auto-corrélation spatiale. Les méthodes de l'économétrie spatiale nous orientent vers une spécification avec erreurs spatialement auto-corrélées qui nous permet de mettre en évidence un effet de débordement géographique. Nous montrons ainsi que le taux de croissance d'une région est influencé positivement par les taux de croissance des régions contiguës.

\section{SUMMARY}

The aim of this paper is to analyze the consequences of spatial dependence on regional growth and the convergence process. Based on a sample of 92 European regions over the 1980-1995 period, we show that the unconditional $\beta$-convergence model is misspecified due to the presence of spatial autocorrelation. Spatial econometric methods lead to a spatial error model. This specification highlights a geographic spillover effect. Indeed, we show that the growth rate of a region is positively influenced by the growth rates of contiguous regions.

\section{NOTES}

(1) MANKIW (1995, p. 304) et TEMPLE (1999, p. 130) attirent également l'attention sur le problème de la dépendance spatiale dans le cadre des modèles de convergence.

(2) LEVINE et RENELT (1992) recensent ainsi dans la littérature plus de 50 variables explicatives et proposent une étude de sensibilité des différentes spécifications envisagées.

(3) Il existe une autre spécificité des données spatiales : l'hétérogénéité spatiale qui n'est pas envisagée ici.

(4) Pour une présentation de ces théories, on peut se référer à FUJTA, THISSE (1997) ou à FUJITA, KRUGMAN, VENABLES (1999).

(5) Tous les calculs ont été effectués en utilisant le logiciel SpaceStat 1.90 élaboré par ANSELIN (1999).

(6) La probabilité critique associée est de 0,85 . 